\title{
Historia del desarrollo de la Didáctica de la Matemática Un estudio realizado con los medios teóricos de la EOS (Enfoque Onto-Semiótico) ${ }^{1}$
}

\author{
Bruno D'Amore ${ }^{12}$ \\ bruno.damore@unibo.it \\ https://orcid.org/0000-0002-5834-9438 \\ Martha Isabel Fandiño Pinilla ${ }^{2}$ \\ https://orcid.org/0000-0002-1592-9918 \\ damore@dm.unibo.it \\ ${ }^{1}$ Universidad Distrital Francisco José de Caldas \\ Bogotá, Colombia; \\ ${ }^{2}$ Universidad de Bologna, NRD clo Departamento de Matemática,
}

Bologna, Italia

Recibido: 12/01/2020 Aceptado: 20/02/2020

\section{Resumen}

En este trabajo se presentan y se sintetizan diversas reflexiones sobre las bases teóricas del Enfoque ontosemiótico del conocimiento matemático y de la enseñanza de la matemática, en el marco general de la progresiva consolidación de la Didáctica de la Matemática como disciplina científica. Se comienza presentando algunas características del conocimiento científico en general, los requisitos que se le exigen a la didáctica para su consideración como ciencia y el problema de la proliferación de marcos teóricos. Seguidamente, se describen algunos rasgos característicos desde el punto de vista ontológico y semiótico como una base constitutiva de la Didáctica de la Matemática. Finalmente se hace referencia a otros marcos teóricos y sus conexiones con el enfoque ontosemiótico.

Palabras clave: bases de la didáctica de la matemática, bases del EOS, relaciones entre teorías, didáctica de la matemática como ciencia.

\section{História do desenvolvimento da Didática da Matemática Um estudo realizado com os médios teóricos do EOS (Enfoque Onto-Semiótico)}

\begin{abstract}
Resumo
Neste trabalho são apresentadas e sintetizadas várias reflexões sobre as bases teóricas do Enfoque Ontosemiótico do Conhecimento Matemática e do Ensino da Matemática (EOS), no marco geral da progressiva consolidação da Didática da Matemática como disciplina científica. O artigo inicia-se apresentando algumas características do conhecimento científico em geral, os requisitos que são exigidos à didática para a sua consideração como ciência y o problema da proliferação de marcos teóricos. Logo, são descritas algumas características do ponto de vista ontológico e semiótico como um alicerce constitutivo da Didática da Matemática. Por fim, se faz referência a outros marcos teóricos e suas conexões como o EOS.

Palavras Chave: Bases da didática da matemática, bases do EOS, relações entre teorias, didática da matemática como ciência.

\footnotetext{
${ }^{1}$ Este artículo hace referencia, analiza y profundiza trabajos previos, en particular: D'Amore (2000, 2001a, 2001b, 2005, 2007); D’Amore, Fandiño Pinilla (2001, 2013); D’Amore, Font, Godino (2007); D’Amore, Godino (2006, 2007; Font, Godino, D’Amore (2007).
} 


\title{
History of the development of the Mathematics Education A study conducted by theoretical means of EOS (Enfoque Onto-Semiotico)
}

\begin{abstract}
In this paper we summary and extend various reflections on the theoretical bases of the ontosemiotic approach to mathematical knowledge and instruction, in the general framework of the progressive consolidation of Didactic of Mathematics as a scientific discipline. We first present some characteristics of scientific knowledge, the requirements for didactics to be considered as a science, and the problem of proliferation of theoretical bases. Then, we describe some characteristic features of the ontological and semiotic point of view, as a constitutive basis for Didactic of Mathematics. Finally, some reference to other theoretical frameworks and their connections with the onto-semiotic approach is made.
\end{abstract}

Keywords: bases of didactic of mathematics, bases of OSA, relations between theories, didactic of mathematics as science.

\section{Breves notas sobre el nacimiento de la Didáctica de la Matemática}

Establecer una fecha de nacimiento para la Didáctica de la Matemática (DdM), es una empresa titánica y sin un éxito garantizado; sin embargo, creemos que muchos concuerdan en afirmar que, para como la entendemos hoy, la DdM como disciplina, nace en los años '70 (Artigue, Gras, Laborde, \& Tavignot, 1984).

Cuando la DdM inició a delinearse, eran tantas sus interpretaciones como cuantos eran los investigadores que declaraban ocuparse de ésta; pero, ya a finales de los años '80, Thomas Romberg, aceptando parámetros “débiles" para la definición de "ciencia consolidada y estable”, declaraba que la DdM podía ser considerada como tal (Romberg, 1988).

Sin embargo, un artículo de Sierpinska y Lerman de 1996 sobre la epistemología de la matemática y sobre la educación matemática evidenciaba una gran cantidad de puntos de vistas teóricos puestos en acto para afrontar la investigación en DdM (Sierpinska, \& Lerman, 1996), puntos de vista que son absolutamente vitales aún hoy, veinte años después.

"Pluralidad de puntos de vista" puede significar "enriquecimiento"; sin embargo, para el progreso de una disciplina y para el potenciamiento de sus aplicaciones prácticas, nos parece inevitable deber cumplir el esfuerzo de identificar pocos conceptos y métodos unificadores que, en un inmediato futuro, nos lleven todos a compartir un verdadero programa de investigación (Lakatos, \& Musgrave, 1960).

\section{Qué entendemos hoy con el término "ciencia"}

Sobre este punto queremos proceder con calma, afrontando el tema desde lejos: nos serviremos principalmente de D’Amore (2007). 
El término "teoría científica" o "ciencia” generalmente se reserva a toda representación ( simbólica, abstracta, escrita, ...) compartida, coherente y plausible, de un conjunto de fenómenos relacionados entre sí por relaciones causales, describibles, significativas (causa efecto, deducción, inducción, abducción, ...) (Flach, \& Hadjiantonis, 2000).

Dejando de lado, por brevedad, el recorrido arcaico de la idea de ciencia, entre las formas actuales de considerar una teoría científica se encuentra la bien conocida y discutida noción de “paradigma” ideada por Thomas Kuhn (Kuhn, 1957); se entiende por "paradigma” el conjunto de las hipótesis teóricas generales y el conjunto de las leyes para sus aplicaciones, aceptadas comúnmente por quienes se declaran pertenecientes a una misma comunidad científica, y que implican un sustancial acuerdo en los juicios profesionales, de mérito y de pertinencia.

En la formación de una nueva comunidad científica, existe un momento a partir del cual se puede hablar precisamente de "paradigma"; la fase precedente está caracterizada por una desorganización, exenta de acuerdos específicos, y con una constante riqueza de debates sobre los fundamentos de la disciplina misma. Como dijimos en precedencia, de forma un poco irónica se puede afirmar que en esta fase hay tantas teorías como investigadores y una continua búsqueda y exigencia de clarificar los puntos de vista propios y las posiciones de los otros. Los trabajos escritos de investigación en este momento están por lo general acompañados de explicaciones sobre las características generales de la investigación misma.

La tesis más famosa de Kuhn (1957) es aquella según la cual el progreso científico procede según "revoluciones", dado que se presenta un cambio, una evolución, sólo después de una crisis.

Otra contribución fundamental fue la que propuso al inicio de los años '60 Imre Lakatos (Lakatos, \& Musgrave, 1960), con la idea de “programa de investigación”, es decir una sucesión de teorías científicas relacionadas entre sí en un desarrollo continuo, que contiene reglas metodológicas de investigación (reglas en positivo, de seguir, reglas en negativo, de evitar). Todo programa debe contener: un núcleo o centro del programa; un sistema de hipótesis auxiliares; una heurística, es decir, los procedimientos que se aplican a la resolución de los problemas. En esta sucesión, una nueva teoría se puede considerar un progreso respecto a la precedente si:

- $\quad$ permite hacer predicciones que la precedente no era en grado de hacer;

- $\quad$ algunas de dichas predicciones se pueden probar como verdaderas; 
- $\quad$ la nueva teoría explica hechos que la precedente no podía probar.

Otra notable contribución teórica fue dada a mitad de los años '80 por Mario Bunge (Bunge, 1985): la ciencia es un cuerpo, en constante crecimiento, de conocimientos, caracterizado por el hecho de tratar conocimientos racionales, sistemáticos, exactos, verificables (por tanto, también falibles).

Los conocimientos científicos coinciden con el conjunto de las ideas sobre un determinado argumento, establecidos provisoriamente en un primer momento; pero después, el concurso de cada uno y el intercambio de informaciones y de ideas da lugar a una comunidad científica. Lo que caracteriza la diferencia entre campos de creencias (religiones, ideologías, políticas, ...) y campos de investigación científica es el tipo de modalidades según las cuales se presentan los "cambios" en las ideas. En los primeros, los cambios se presentan a causa de "revelaciones", controversias, presiones sociales; en los segundos los cambios son continuos a raíz de los resultados de la investigación misma.

Según exigencias "débiles”, una teoría científica se define hoy como tal cuando dispone de un objeto específico de estudio, de un método de investigación propio y de un lenguaje específico compartido; a esta exigencia hacen referencia los teóricos de las ciencias humanas, para llamar "ciencias" precisamente, dichos dominios de estudio.

Esta exigencia “débil” permitió, en las últimas décadas, la proliferación del apelativo de "ciencia" dado a muchas disciplinas. De hecho, cualquier disciplina a cuyo desarrollo concurran estudiosos que se reconozcan y se acepten recíprocamente como expertos de ésta, fundando una comunidad de prácticas compartidas y que hacen uso del mismo lenguaje, antes o después adquiere precisamente las características descritas líneas arriba.

El problema de la posible repetición de los experimentos, de la correcta definición de las variables en juego, del sentido que adquieren términos como "riguroso", "verdadero", etc., tiende a desvanecer o, mejor, a sufrir profundas modificaciones.

Lo que existe de común en todas estas interpretaciones es que las teorías científicas no pueden ser creaciones o invenciones de una única persona, sino que debe existir una comunidad de personas entre las cuales rige un sustancial acuerdo sea sobre los problemas significativos de la investigación, sea sobre las modalidades en las cuales dicha investigación se explica, sea sobre el lenguaje usado. 


\section{La DdM es una ciencia}

Entrando un poco más en la dirección precedentemente delineada, Romberg (1988), para definir las características peculiares de una teoría científica consolidada y estable, afirmaba explícitamente que:

- debe existir un conjunto de investigadores que demuestren interés en aspectos comunes; en otras palabras, deben existir problemáticas centrales que guían el trabajo de los investigadores y que estas problemáticas son compartidas;

- las explicaciones dadas, a estas problemáticas, por los investigadores deben ser del tipo causal;

- el grupo de investigadores debe haber elaborado un vocabulario y una sintaxis común, con la cual el grupo se manifiesta de acuerdo;

- el grupo debe haber elaborado procedimientos propios para aceptar o rechazar los enunciados en una manera considerada por sus miembros objetiva $\mathrm{y}$ ampliamente compartida.

Entre las ciencias así entendidas, se incluyen las didácticas disciplinarias y en particular la DdM:

- es evidente la existencia de un numeroso grupo internacional de investigadores en las diversas didácticas disciplinarias que tienen intereses comunes,

- para quienes existen problemáticas consideradas centrales y compartidas,

- que dan (desde hace unas cuatro décadas) explicaciones de carácter causal,

- que han elaborado un vocabulario común, siempre más compartido.

Estos investigadores tienen congresos y revistas específicas, al interior de las cuales las propuestas de comunicaciones o de publicaciones son analizadas sobre la base de procedimientos ampliamente compartidas. Estamos, por tanto, plenamente en las condiciones propuestas por Romberg para poder afirmar que muchas didácticas disciplinarias tienen todas las características para poder ser consideradas ciencias consolidadas y estables.

Además: nos parece necesario e inevitable que quien se ocupa de DdM deba ser óptimo conocedor de la matemática; el hecho es que no se puede ni pensar de entender problemas de falta de comprensión del concepto de límite (por ejemplo) por parte de un estudiante, si quien cumple este análisis no domina él mismo el concepto de límite. No solo conoce la matemática 
necesaria para entender esta noción, pero la conoce profundamente desde un punto de vista histórico-crítico, epistemológico, didáctico, hasta hacerla propia, es decir, no solo una noción aprendida en cursos, sino una "construcción personal", elaborada en propio.

Por tanto, el estudio de la DdM y aún más la investigación en este campo requiere una fuerte interrelación de base sobre la competencia en matemática por parte de quien la realiza (este tema es mucho más estudiado y analizado en Fandino Pinilla, 2011, texto al cual reenviamos). ${ }^{2}$

Desde hace tiempo sugerimos que se puede interpretar la DdM como una disciplina interna a la matemática misma, una matemática aplicada, precisando aún más: aplicada a la problemática de la enseñanza - aprendizaje de la matemática. Nos parece que esta pertenencia sea cónsona, lógica, adecuada, funcional que no la de presentar la DdM dentro del ámbito de las ciencias de la formación o de la didáctica general (D’Amore, \& Frabboni, 1996; 2005; D’Amore, \& Fandiño Pinilla, 2007, 2020).

Consideramos oportuno y genial que, el 6 de julio de 2006, en el congreso Joint Meeting of UMI-SIMAI/SMAI-SMF: Mathematics and its Applications, que se llevó a cabo en el Departamento de Matemática de la Universidad de Turín, Italia, se haya incluido como temática precisamente la DdM con un amplio Panel on Didactics of Mathematics. Y que las actas de aquel panel hayan sido publicadas sobre una revista de DdM (AA. VV., 2007).

Volviendo, por tanto, a la teoría de una didáctica disciplinar, debemos en primer lugar hacer el esfuerzo de afrontar los problemas meta-disciplinarios; para nosotros, entre éstos, está la urgente necesidad de clarificar las nociones teóricas que se utilizan en DdM, en particular las nociones usadas para analizar los fenómenos del aprendizaje y la cognición.

Sobre este tema, no existe un consenso incluso al interno de aquella corriente que se suele 1lamar “epistemológica” o “didáctica fundamental” (Brousseau, 1989; Gascón, 1998). Para dar cuenta de lo que estamos afirmando, es suficiente observar la variedad de nociones (y de sus interpretaciones) que son usadas sin que haya habido un análisis previo, una confrontación, una clarificación, una limpieza.

\footnotetext{
${ }^{2}$ Esto no excluye que en el pasado y/o en la actualidad encontremos estudiosos de otras disciplinas, no matemáticos de profesión, que aportaron o estén aportando a la investigación en DdM una contribución extraordinaria; pero ciertamente esto es un hecho inusitado. Queremos recordar explícitamente a tres psicólogos que ayudaron y siguen ayudando a crear las bases mismas de la DdM: Efraím Fischbein, Gérard Vergnaud y Raymond Duval.
} 
Entre los términos que consideramos mayormente abusados, encontramos: conocimiento, saber, concepciones, conceptos, esquemas, invariantes operatorias, significado, praxeología, ... Se trata de nociones de base, de los instrumentos, de las herramientas, cada uno con potencialidades y límites, según la interpretación que de éstos se haga.

El problema interesante que se delineó en los últimos veinte años fue el de la necesidad de la elaboración de nuevos constructos cognitivos que superen eventuales limitaciones de aquellos existentes, utilizados a veces de forma acrítica, partiendo de lo que tenemos actualmente a disposición. Sólo así será posible una operación fundamental de reconocimiento de concordancias, complementariedades, redundancias, discordancias, ... entre las diversas posiciones.

En particular, si el objetivo fuese el de interpretar la DdM como una matemática aplicada, es evidente que cada término debería ser definido de forma unívoca y precisa. La tarea parece gravosa, pero, la matemática afrontó en precedencia ese tipo de dificultades, logrando dar definiciones de ideas que parecían escapar a todo intento de clasificación terminológica; pensemos, por ejemplo, en la idea de infinito que necesitó más de dos milenios de intentos (D’Amore, 1996a; 1996b).

El uso del término "cognitivo", por ejemplo, es en sí mismo conflictual. Se encuentra en varias ocasiones para indicar conocimientos subjetivos, pero en ocasiones también para procesos mentales que las personas ponen en acto en el momento de afrontar un problema.

Desde el punto de vista psicológico de la cognición matemática, dichos procesos mentales, que tienen lugar en el cerebro de los seres humanos, son los únicos constituyentes del conocimiento que deben ser considerados. Pero esta afirmación limitativa no tiene en cuenta el hecho que los sujetos dialogan entre sí, encontrando o buscando acuerdos, regulan las formas de expresión y de actuación frente a determinadas clases de problemas; y que de estos "sistemas de prácticas compartidas" emergen objetos institucionales los cuales, a su vez, condicionan las formas de pensar y de actuar de los miembros de dichas instituciones.

Por tanto, junto a los conocimientos subjetivos que emergen de las formas de pensar y de actuar de los sujetos entendidos de forma individual, es necesario considerar los conocimientos institucionales, a los cuales se debe atribuir un cierto grado de objetividad. 
Esto lleva inmediatamente a distinguir, en la cognición individual y en la cognición general, la dualidad "cognición individual" - "cognición institucional", entre las cuales se instauran relaciones dialécticas complejas.

Cognición individual: resultado del pensamiento y de la acción de un sujeto pensado como individuo frente a una cierta clase de problemas;

Cognición institucional: resultado del diálogo, del acuerdo y de la regulación de las acciones al interior de un grupo de individuos, frente a una cierta clase de problemas.

Una cognición individual no necesariamente coincide con la cognición institucional; se puede identificar la cognición personal con el término "cognitivo", como se hace in psicología cognitiva (Neisser, 1967); y la cognición institucional con el término "epistémico", dado que se ocupa de un conocimiento institucional.

Esta distinción es necesaria para afrontar dos puntos de vista de la investigación, el punto de vista antropológico (Chevallard, 1992; 1999) y el punto de vista ontosemiótico (Godino. \& Batanero, 1994; Godino, 2002).

No trataremos aquí el punto de vista antropológico, reenviando al análisis detallado propuesto en D’Amore y Godino (2006).

\section{Interpretación de las bases constitutivas de la DdM desde los puntos de vista ontológico y semiótico}

Las aplicaciones de los supuestos ontológicos de la semántica realista a la matemática nos llevan a una visión platónica de los objetos matemáticos (conceptos, proposiciones, teorías, contextos, ...) (D’Amore, \&Godino, 2006). Según esta posición filosófica, las nociones y las estructuras matemáticas tienen una existencia real, independiente del ser humano y de sus actividades, privadas o sociales, en algún dominio real. El conocimiento matemático consiste en descubrir relaciones pre-existentes que relacionan entre sí dichos objetos.

Dicha concepción implica además una visión absolutista del conocimiento matemático, en el sentido que es considerado como un sistema de verdades seguras, ciertas e inmutables. Con este presupuesto, por ejemplo, el significado del término "función" coincide con el concepto de función delineado, explicitado, externalizado por una oportuna y "correcta" definición matemática.

Desde un punto de vista epistemológico, la definición pragmatista del significado es 
[...] mucho más satisfactoria que aquella dada al interior de la teoría realista: con la desaparición de los conceptos y de las proposiciones como datos independientes de la lengua, se disipa también el problema de cómo puedan ser conocidas estas entidades, y nos acercamos a los fenómenos que justifican la dependencia del pensamiento y de la experiencia respecto al lenguaje (Kutschera, 1979; p. 148).

Desde nuestro punto de vista, los supuestos ontológicos del constructivismo social como filosofía de la matemática (Ernest, 1998) implican, entre otras cosas, la adopción de las teorías pragmatistas del significado. Los objetos matemáticos deben/pueden ser considerados como símbolos de unidades culturales, emergentes de un sistema de usos ligados a las actividades matemáticas que realizan grupos de personas y que, por tanto, evolucionan con el transcurrir del tiempo.

En nuestra concepción, lo que determina el emerger progresivo de los “objetos matemáticos" es el hecho de que, en el seno de ciertas instituciones, se realizan determinados tipos de prácticas y que el "significado" de dichos objetos está íntimamente relacionado con los problemas afrontados y con las actividades realizadas por los seres humanos, no pudiéndose reducir el significado del objeto matemático a su mera definición matemática. La definición sintetiza un significado que es debido a la actividad, no es el significado o su expresión unívoca.

Siguiendo lo dicho hasta ahora, tomemos en examen las consideraciones de Ullmann (1978) que abren el camino a las siguientes prospectivas: las posiciones realistas y pragmatistas no son contradictorias; por tanto, la posición antropológica no está en contradicción con la realista.

En Ullman las teorías realistas son llamadas "referenciales" mientras las pragmatistas son llamadas "operacionales o contextuales"; desde su punto de vista, las teorías pragmatistas son un complemento de las teorías realistas:

El significado de una palabra se puede verificar sólo estudiando su uso [y aquí nos recuerda Wittgenstein]. No existe ningún atajo hacia el significado mediante la introspección o algún otro método. El investigador debe, en primer lugar, organizar una muestra adecuada de contextos y afrontarlos con un espíritu abierto, permitiendo que el significado o los significados emerjan de los contextos mismos. Una vez concluida esta fase, se puede pasar a la fase "referencial" y formular el significado o los significados evidenciados de esta forma. (Ullman, 1962, p. 76). 
Puede ser iluminante en este punto citar la llamada "máxima pragmática" de Peirce: «Considerar cuáles son los efectos prácticos que nosotros pensamos puedan ser productos del objeto en nuestra concepción. La concepción de todos estos efectos es la concepción completa del Objeto» (Peirce, CP, 5.18, en Pierce, 1960). [Traducción nuestra].

Volvamos a Ullman:

La relación entre los dos métodos o, mejor, entre las dos fases de la investigación, es, en definitiva, la misma que se tiene entre el idioma y su expresión oral: la teoría operacional trata del significado en el hablado; aquella referencial, del significado en el idioma. No existe, absolutamente, necesidad de colocar las dos formas de acceso en oposición, uno frente al otro: cada uno conduce a su propio lado del problema y ninguno está completo sin el otro. (Ullman, 1962, pp. 7677)

Recogiendo esta significativa observación de Ullman, y volviendo al campo que nos interesa, creemos poder afirmar que el significado de los objetos matemáticos inicia como pragmatista, relativo al contexto; pero, entre los tipos de uso relativos a dicho significado, existen algunos que permiten orientar los procesos de enseñanza - aprendizaje de la matemática. En la Didáctica de la Matemática, estos tipos de usos se objetivan a través del lenguaje y terminan constituyendo referencias específicas del léxico institucional.

Lo aquí delineado es el punto de partida de una visión de la DdM que amplía el punto de vista antropológico, elimina los límites, se acerca a la "práctica" compartida en aula, supera la supuesta dicotomía entre realismo y pragmatismo, así como entre antropología y psicología.

\section{Los marcos teóricos en DdM como desarrollo de diversas teorías}

Una de las tareas de mayor urgencia e importancia que deben ser afrontadas por los investigadores en DdM, como habíamos sugerido precedentemente (en 1.), es la clarificación, la comparación y la articulación de los marcos teóricos que se están usando actualmente. El

problema es urgente y crítico, dado que, nuestra disciplina está ligada a otras, como la epistemología, la psicología, la matemática, la semiótica etc., y se están usando instrumentos y presupuestos teóricos divergentes, cuya coherencia y utilidad no es de hecho obvia y no puede ser dada por descontada.

En el actual panorama de la DdM observamos un cierto absolutismo teórico (cerrado en sí mismo) y una desarticulación conceptual y metodológica. Este problema se observa no sólo entre paradigmas y escuelas de pensamiento lejano (pragmatismo, realismo, constructivismo, 
cognitivismo, etc.), sino incluso dentro de las teorías emergentes de nivel intermedio que comparten un mismo paradigma epistemológico de base.

Por ejemplo, en el caso de la TAD, ¿qué relaciones existen con la teoría de las situaciones de Brousseau, o con la teoría de los campos conceptuales de Vergnaud, o con la teoría de la dialéctica instrumento - objeto de Douady? ¿Qué relaciones existen entre estas teorías y la teoría de los registros de representación semiótica de Duval? ¿O con la teoría APOS de Dubinsky? Etc.

Para poder hacer estas comparaciones y estas articulaciones es necesario construir un sistema de referencia global que permita situar cada una de las teorías en el panorama integral de la DdM. Es necesario tener presente simultáneamente las diferentes dimensiones implicadas en los problemas de enseñanza y de aprendizaje de la matemática (dimensión epistémica, cognitiva, instruccional, política, etc.) y de los diversos niveles de análisis.

El punto de vista ontosemiótico (EOS) nació alrededor del 1994 (Godino y Batanero, 1994) con el objetivo de iniciar este camino de reflexión meta-didáctica, partiendo de la constatación de algunos límites en el punto de vista antropológico que había iniciado a formular Chevallard (1991; 1992). En particular, se trató de rectificar la elección anti-psicológica inicial, que se consolidó en los trabajos sucesivos, y su divergencia de otras teorías precedentes, como la teoría de las situaciones o aquella de los campos conceptuales (Vergnaud, 1990).

Pero no podemos olvidar el recorrido, el largo recorrido teórico de la DdM y de las teorías que decretaron su surgimiento y después la aceptación como teoría científica (D’Amore, \& Fandiño Pinilla, 2013).

La teoría de las situaciones didácticas fue la primera en nacer; todos nosotros, investigadores en DdM, ya veteranos, fuimos fascinados por su forma de entender el aprendizaje; era la primera vez que los matemáticos hacían reflexiones de este tipo y en esta dirección.

Pero después la DdM evolucionó, y surgieron otras tantas teorías, inútil hacer una lista aquí: citaremos algunas líneas abajo. Muchas de estas teorías son evoluciones de la teoría de las situaciones, otras examinan cuestiones diferentes; algunas nacieron y murieron, otras se desarrollaron de forma impensable.

Las teorías nacen y mueren, se puede establecer un contraste entre ellas o buscar conexiones entre estas o incluso hacer que colimen incluyéndolas en otras teorías. Son muchos 
los estudios en esta dirección; nosotros nos limitamos reenviar a: Prediger, Bikner-Ahsbahs y Arzarello (2008), Radford (2008a; b; 2011), Bikner-Ahsbahs, Dreyfus, Kidron, Arzarello, Radford, Artigue y Sabena (2010).

Pero las teorías nuevas nacen con objetivos bien precisos, no sólo para absorber o incluir las teorías precedentes, sino también para estudiar factores que a las precedentes se les escapaban o para estudiar hechos que a las precedentes no les interesaban (D'Amore, 2007).

Así, teorías construidas sucesivamente a la teoría de las situaciones tuvieron objetivos diversos, fueron bien aceptadas en el panorama de los investigadores internacionales, pero no sustituyen la teoría de situaciones porque estas nuevas teorías tienen objetivos diversos.

Por ejemplo, la teoría APOS (que describe cómo las Acciones se interiorizan en Procesos que después son encapsuladas como Objetos mentales, que toman su puesto en más sofisticados Esquemas cognitivos) (Tall, 1999), creada por Ed Dubisky en los años '80, tuvo un gran éxito internacional (y también grandes críticas), pero entre sus objetivos no estaba el de entender las situaciones de aula, como sí lo logra hacer la teoría de las situaciones (Dubinsky, 1991a, b).

Por ejemplo, el gran aparato introducido por Raymond Duval en 1993 para mostrar cómo las actividades de enseñanza - aprendizaje de la matemática en aula están fuertemente conexas con las tres acciones cognitivas de representar, tratar, convertir típicas de la semiótica, trajo a nuestra disciplina excelentes resultados, pero nada tiene que ver con las descripciones que la teoría de las situaciones nos enseña a observar y reconocer (Duval, 1993;1995). ${ }^{3}$

Por ejemplo, la teoría EOS (Enfoque Onto Semiótico) tuvo un gran éxito internacionalmente desde cuando fue creada en los años '90 por uno de los grupos de investigación con sede en la Universidad de Granada, el grupo dirigido por Juan D. Godino; dicha teoría engloba en cierto sentido la llamada TAD (teoría antropológica de la didáctica) creada por Yves Chevallard en los primeros años '90, pero con objetivos declarados manifiestamente diversos de los objetivos perseguidos por la teoría de situaciones (Chevallard, 1991, 1992; D’Amore, \& Godino, 2006; 2007; Font, Godino, \& D’Amore, 2007), aún sin estar en antítesis y teniendo importantes puntos de comunión, como veremos en este mismo artículo.

Por ejemplo, la teoría semiótico cultural de Luis Radford tiene la capacidad de explicar modalidades de aprendizaje relacionadas con actividades semióticas por parte de estudiantes,

\footnotetext{
3 En verdad, las cosas no son así totalmente; para un intento de consolidación entre estas dos teorías, véase: D’Amore (2003).
} 
por ejemplo, en el aprendizaje de la generalización o del álgebra precoz, que ninguna otra teoría precedente tiene. Pero no incluye el estudio general de las situaciones de aula, las cuales, dentro de esta teoría, son aceptadas como elementos normativos. Un análisis comparativo entre teoría de las situaciones y teoría de la objetivación se puede ver en Asenova, D’Amore, Fandiño Pinilla, Iori y Santi (2020).

Indudablemente se trata de una de las teorías que, más que ninguna otra, cambió nuestra actitud frente al aprendizaje y a la DdM; en pocos años se impuso con fuerte sustentamiento que nos sorprende a todos, tanto, que no es fácil reconstruir su historia (cosa que nosotros hicimos, incluso pidiendo ayuda al mismo creador: Radford, 1998, 2000a, b, 2001, 2003, 2006a, b; véase: D’Amore, \& Radford, 2017).

Y así sucesivamente, podríamos continuar citando teorías que siguieron a la teoría de situaciones, con sus características innovadoras y funcionales, a veces sólo descriptivas, a veces operativas.

Ahora bien, la actitud que se encuentra en algunos centros de investigación y en algunos investigadores y docentes de DdM de opacar las primeras teorías en favor de las nuevas es, por decir poco, ridículo. Uno de los artículos de Radford, enviado para su publicación en 1998 salió sólo en el 2003 porque en aquel tiempo algunas revistas de didáctica rechazaban sin apelación los artículos que hablaban de semiótica. Debemos decir que, aún en 2005, uno de los autores de este artículo encontró cierta dificultad para publicar un artículo porque, según uno de los árbitros que lo leyó, "se cita demasiadas veces a Raymond Duval” (conservamos esta carta). ${ }^{4}$

Pero esto es lo que sucede siempre a los anticipadores; Guy Brousseau inició a elaborar su teoría, que después tuvo fama internacional, en los años ' 60 y, en los años '70, ya era madura; pero tuvo que esperar hasta 1986 para ver publicado su famoso artículo, tal vez el más citado en el mundo en nuestro sector (Brousseau, 1986).

El hecho es que en los años '60 y '70 las revistas de, digamos así, enseñanza de la matemática, rechazaban estos artículos considerados "bizarros y extraños" como eran calificados los escritos por Brousseau; ¡tan es así que tuvo que publicar un artículo sobre una Revue de laryngologie, otologie, rinologie (Brousseau, 1980)! En aquellos tiempos, los nombres que dictaban ley eran aquellos de Zoltan Dienes, Georges y Frédérique Papy, ... quienes, más que teorías, proponían sistemas de enseñanza a veces bizarros basados en sus propias intuiciones

\footnotetext{
${ }^{4}$ El artículo salió de todas formas en 2006.
} 
y sin bases científicas. Los estudios de Brousseau dedicados a demoler duramente estos enfoques de enseñanza de la matemática son bien conocidos y tuvieron un éxito evidente: ¿Quién recuerda hoy estos nombres? Sin una teoría científicamente basada en enfoques epistemológicos sensatos y bien fundamentados, estos episodios están condenados a desaparecer.

Pero las teorías sólidas, aquellas que dan resultados, aquellas que permiten entender la actitud de los estudiantes y de los docentes en las situaciones de aula, no son olvidadas. Es más, deben ser colocadas en la base, al inicio, de cualquier curso que pueda servir a quien de estas teorías debe hacer uso concreto en aula, los docentes, y a los futuros investigadores (estamos hablando, respectivamente, de cursos para docentes en formación inicial o en servicio, y de cursos de maestría y de doctorado). De lo contrario, un día, alguien creerá descubrir aquellas cosas, ignorando que ya habían sido estudiadas, dará a estos estudios un nombre nuevo, creyendo de estar proponiendo un avance en el estudio de la didáctica de la matemática. Lo cual sería bastante ridículo.

Sería como re-descubrir que existen fórmulas generales que usan sólo operaciones racionales y la extracción de raíz cuadrada para encontrar las raíces de las ecuaciones algebraicas generales de III grado con coeficientes enteros o racionales, con buena paz de Tartaglia (Tartamudo) y Cardan.

\section{Relaciones del EOS con la teoría de las situaciones}

El objetivo de continuar en la comparación y en la articulación de diferentes modelos teóricos, llevaron al EOS a formular algunas "nociones primitivas" con un alto grado de generalidad, como son "práctica matemática”, “institución”, “objeto matemático”, "función semiótica" y las dualidades cognitivo-antropológico (persona-institución, elemental-sistémico, ostensivo-no ostensivo, extensiva-intensiva, expresión-contenido). Estos instrumentos ofrecen una plataforma unificada a partir de la cual es posible afrontar las ya recordadas tareas de comparación y de articulación de los marcos teóricos usados en DdM.

Muchas de las propuestas estrictamente didácticas desarrolladas en los años dentro del EOS son compatibles totalmente con las bases de la teoría de las situaciones como se ve explícitamente enunciado en Godino y Batanero (2016):

La teoría de las situaciones didácticas formulada por Brousseau constituye, desde nuestro punto de vista, una teoría del aprendizaje organizado de la matemática, es decir, una teoría de la 
enseñanza de la matemática, en acuerdo con los presupuestos epistemológicos y psicológicos evidenciados en precedencia. Describe un ambiente de aprendizaje potente en el cual no sólo se presta atención al saber matemático puesto en juego en la propuesta de trabajo sino también a las actividades de comunicación en aula, todo esto en una secuencia ordenada de situaciones didácticas. (...) la teoría de las situaciones didácticas [y aquí se citan los trabajos de G. Brousseau] que nos sirve de referencia evidencia el papel de las situaciones de acción para hacer que los estudiantes den sentido a las nociones y a los procesos de la matemática.

Efectivamente, algunos de los presupuestos de base de las dos teorías son similares:

- la elección de situaciones de aprendizaje significativas preparadas por el docente;

- el papel del docente como un director (de teatro) de la actividad de los estudiantes implicados en situaciones a-didácticas;

- el papel importante del conocimiento matemático (el Saber) para poder proceder a la transposición didáctica;

- la importantísima fase de la institucionalización que, en la teoría de las situaciones, es aquella final en el uso de situaciones a-didácticas;

- etc.

En D’Amore, Font y Godino (2007) se muestra cómo, con instrumentos del EOS y de la sociología, es posible evidenciar cómo el fenómeno del contrato didáctico, introducido por la teoría de las situaciones de Guy Brousseau, puede tener explicaciones de carácter sociológico. De otra parte, estos aspectos habían sido puestos en evidencia en D’Amore (2005) y en Bagni y D’Amore (2005).

Como podemos ver la red constructiva entre teorías es decididamente fuerte y significativa.

\section{Conclusiones}

Como en toda ciencia consolidada, también en DdM existen desarrollos teóricos, procesos de desarrollo, arrestos momentáneos e ideas brillantes que permiten reflexiones críticas maduras.

Pensamos que el EOS es una de aquellas teorías que determinaron el nacimiento de formas nuevas de pensamiento y de reflexión en el panorama de la DdM en el mundo. Sin embargo, aún hoy, si se desea describirla y evidenciar sus bases filosóficas, se debe hacer 
referencia a teorías precedentes que indudablemente fueron las bases para esta innovadora creación. Nada nace de la nada y las "espaldas de los gigantes" que nos precedieron están siempre ahí, listas para fungir de apoyo a nuevos investigadores de poco legados al litoral del mar a contemplar su vastedad.

Nosotros somos partidarios de la necesidad, en los límites de lo posible (en realidad: más allá de estos límites), de estudiar siempre la posibilidad de la unificación de las teorías o por lo menos su correlación sistematizada; casi nunca nos ha sucedido de tener que aceptar una falta total de nexos o constituyentes comunes entre dos teorías, por muy distantes que aparezcan a simple vista.

\section{Referencias}

AA. VV. (2007). Actas del: Joint Meeting of UMI-SIMAI/SMAI-SMF: Mathematics and its Applications. Panel on Didactics of Mathematics. Departamento de Matemática, Universidad de Turín, 6 julio 2006. La matematica e la sua didattica, 21(1).

Artigue, M., Gras, R., Laborde, C., \& Tavignot, P. (1984). Vingt ans de didactique des mathématiques en France. Grenoble: La pensée sauvage.

Asenova, M., D’Amore, B., Fandiño Pinilla, M. I., Iori, M., \& Santi, G. (2020). La teoria dell'oggettivazione e la teoria delle situazioni didattiche: Un esempio di confronto tra teorie in didattica della matematica. La matematica e la sua didattica, 28(1). En curso de impresión.

Bagni, G. T., \& D’Amore, B. (2005). Epistemologia, sociologia, semiotica: la prospettiva socioculturale. La matematica e la sua didattica, 19(1), 73-89.

Blumer, H. (1969). Symbolic interactionism. Perspective and method. Englewood Cliffs, NJ: Prentice Hall.

Bikner-Ahsbahs, A., Dreyfus, T., Kidron, I., Arzarello, F., Radford,L., Artigue, M., \& Sabena, C. (2010). Networking of Theories in Mathematics Education. En: M. F. Pinto M.F. y T. F. Kawasaki (Compiladores). Proceedings of the 34th Conference of the International Group for the Psychology of Mathematics Education. Vol. 1, 145-175. Bello Horizonte, Brasil: PME.

Brousseau G. (1980). Les échecs électifs dans l'enseignement des mathématiques à l'école élémentaire. Revue de laryngologie, otologie, rinologie, 101(3-4), 107-131.

Brousseau, G. (1986). Fondements et Méthodes de la Didactique des Mathématiques. Recherches en didactique des mathématiques, 7(2), 33-115.

Brousseau, G. (1989). La tour de Babel. Études en didactiques des mathématiques. 2. Irem de Bordeaux.

Bunge, M. (1985). Pseudociencia e ideología. Madrid: Alianza. 
Chevallard, Y. (1991). Dimension instrumentale, dimension sémiotique de l'activité mathématique. Séminaire de Didactique des Mathématiques et de l'Informatique de Grenoble. LSD2, IMAG, Université J. Fourier, Grenoble.

Chevallard, Y. (1992). Concepts fondamentaux de la didactique: perspectives apportées par une approche anthropologique. Recherches en Didactique des Mathématiques, 12 (1), 73-112.

Chevallard, Y. (1999). L'analyse des pratiques enseignantes en théorie anthropologique du didactique. Recherches en Didactique des Mathématiques, 19 (2), 221-266.

D’Amore, B. (1996a). L'infinito: storia di conflitti, di sorprese, di dubbi. Un fertile campo per la ricerca in Didattica della Matematica. La matematica e la sua didattica, 10(3), 322-335. [Este texto fue republicado en idioma español, con bibliografía completa: El infinito: una historia de conflictos, de sorpresas, de dudas. Epsilon, 12(36), 1996, 341-360. Este artículo fue republicado, con bibliografía completa en: Bollettino dei docenti di Matematica, 17(33), 1996, 1-10].

D'Amore, B. (1996b). Infinite processes throughout the curriculum. Proceedings of the $8^{\text {th }}$ ICME International Congress on Mathematical Education. Sevilla 14-21 Julio 1996, 309311. (Se trata de la conferencia inaugural del Topic Group del cual el autor era el Chief Organizer).

D’Amore, B. (1996c). Elementi di didattica della matematica. Prefacio de Colette Laborde. Bologna: Pitagora. [Versión en idioma español ampliada y actualizada: 2006, Didáctica de la matemática. Prefacios de Colette Laborde, Guy Brousseau y Luis Rico Romero. Bogotá: Magisterio]. [Versión en idioma portugués ampliada y actualizada: 2007, Elementos de didáctica da matemática. Prefacios de Colette Laborde, Guy Brousseau, Luis Rico Romero y Ubiratan D’Ambrosio. São Paulo: Livraria da Física].

D’Amore, B. (2000). "Concetti" e "oggetti” in Matematica. Rivista di Matematica dell'Università di Parma, 6(3), 143-151.

D’Amore, B. (2001a). Conceptualisation, registres de représentations sémiotiques et noétique: interactions constructivistes dans l'apprentissage des concepts mathématiques et hypothèse sur quelques facteurs inhibant la dévolution. Scientia Paedagogica Experimentalis [Gent, Bélgica], 38(2), 143-168.

D’Amore, B. (2001b). Un contributo al dibattito su concetti e oggetti matematici: la posizione "ingenua" in una teoria "realista" vs il modello "antropologico" in una teoria "pragmatica". La matematica e la sua didattica, 15(1), 4-30.

D’Amore, B. (2001c). Scritti di Epistemologia matematica. 1980-2001. Bologna: Pitagora.

D’Amore, B. (2003). Le basi filosofiche, pedagogiche, epistemologiche e concettuali della Didattica della Matematica. Prefacio de Guy Brousseau. Bologna: Pitagora. [Versión en idioma español: D’Amore, B. (2005). Bases filosóficas, pedagógicas, epistemológicas y conceptuales de la Didáctica de la Matemática. Prefacios de Guy Brousseau y Ricardo Cantoral. México DF, México: Reverté-Relime. Traducción de Martha Isabel Fandiño Pinilla].

D’Amore, B. (2005). Pratiche e metapratiche nell'attività matematica della classe intesa come società. Alcuni elementi rilevanti della didattica della matematica interpretati in chiave sociologica. La matematica e la sua didattica, 19(3), 325-336. 
D’Amore, B. (2007). Voces para el diccionario: Frabboni F., Wallnöfer G., Belardi N., Wiater W. (Compiladores) (2007). Le parole della pedagogia. Teorie italiane e tedesche a confronto. Turín: Bollati Boringhieri. Voces: Didattica disciplinare (72-75), Formazione in scienze naturali (140-142), Formazione in matematica (145-147), Scienza (335-337).

D’Amore, B., \& Fandiño Pinilla, M. I. (2001). Concepts et objets mathématiques. En: Gagatsis A. (compilador.) (2001). Learning in Mathematics and Sciences and Educational Technology. Vol. 1. Nicosia: Intercollege Press. 111-130.

D’Amore, B., \& Fandiño Pinilla, M. I. (2007). Le didattiche disciplinari. Prefacio de Franco Frabboni. Trento: Erickson.

D’Amore, B., \& Fandiño Pinilla, M. I. (2013). Il passo più lungo. Sulla necessità di non buttare a mare (in nome di un vacuo modernismo) teorie di didattica della matematica che spiegano, in maniera perfetta, situazioni d'aula reali. Bollettino dei docenti di matematica, [Bellinzona, Suiza], 34(66), 43-52.

D’Amore, B., \& Fandiño Pinilla, M. I. (2020). Per una teoria delle didattiche disciplinari. Saggio per docenti e ricercatori. Prefazione di Maura Iori. Bologna: Pitagora.

D’Amore, B., Fandiño Pinilla, M. I., Marazzani I., \& Sarrazy, B. (2018). El contrato didáctico en Educación Matemática. Prólogo y epílogo de Guy Brousseau. Bogotá: Magisterio. [Edicion en italiano: D’Amore, B., \& Fandiño Pinilla, M. I., \& Marazzani, I., \& Sarrazy, B. (2020). Gli effetti del contratto didattico in aula. Uno strumento concreto per gli insegnanti di Matematica. Prefacio y posfacio de Guy Brousseau. Bologna: Pitagora].

D’Amore, B., Font, V., \& Godino, J. D. (2007). La dimensión meta-didáctica en los procesos de enseñanza y aprendizaje de la matemática. Relime, 38(2), 49-77.

D’Amore, B., \& Frabboni, F. (1996). Didattica generale e didattiche disciplinari. Milán: Angeli.

D’Amore, B., \& Frabboni, F. (2005). Didattica generale e didattica disciplinare. Milán: Bruno Mondadori.

D’Amore, B., \& Franchini, D., \& Gabellini, G., \& Mancini, M., \& Masi, F., \& Matteucci, A., \& Pascucci, N., \& Sandri, P. (1995). La ri-formulazione dei testi dei problemi scolastici standard. L'insegnamento della matematica e delle scienze integrate, 18 A (2), 131-146. [Este artículo fue republicado en idioma inglés en: A. Gagatsis y L. Rogers (Compiladores) (1996), Didactics and History of Mathematics. Erasmus ICP 954 G 2011/11. Thessaloniki. 53-72].

D’Amore, B., \& Godino, D. J. (2006). Punti di vista antropologico ed ontosemiotico in Didattica della Matematica. La matematica e la sua didattica, 21(1), 9-38.

D’Amore, B., \& Godino, D. J. (2007). El enfoque ontosemiótico como un desarrollo de la teoría antropológica en Didáctica de la Matemática. Relime, 10(2), 191-218.

D’Amore, B., \& Radford, L. (2017). Enseñanza y aprendizaje de las matemáticas: problemas semióticos, epistemológicos y prácticos. Bogotá: Editorial Universidad Distrital Francisco José de Caldas.

D’Amore, B., \& Sandri, P. (1993). Una classificazione dei problemi cosiddetti impossibili. La matematica e la sua didattica, 16(3), 344-346. [Este artículo fue republicado en: A. 
Gagatsis (Compilador) (1994), Didactiché ton Mathematicon. Erasmus ICP 93G 2011/II. Thessaloniki. 247-252 (en griego), 579-584 (en francés). Este artículo fue además restampado en: Cahiers de Didactique des Mathématiques. 16-17, Junio 1995, 11-28 (en griego), 103-110 (en francés)].

Dubinsky, E. (1991a). Reflective Abstraction in Advanced Mathematical Thinking. En D. Tall (Compilador.), Advanced Mathematical Thinking (pp. 95-126). Dordrecht, The Netherlands: Kluwer.

Dubinsky, E. (1991b). The Constructive Aspects of Reflective Abstraction in Advanced Mathematics. En L. P. Steffe (Compilador.), Epistemological Foundations of Mathematical Experiences, (pp. 160-202). New York: Springer-Verlag.

Duval, R. (1993). Registres de représentations sémiotique et fonctionnement cognitif de la pensée. Annales de Didactique et de Sciences Cognitives [ULP, IREM Strasbourg], 5(5), 37-65.

Duval, R. (1995). Sémiosis et pensée humaine. Registres sémiotiques et apprentissages intellectuels. Berne: Peter Lang.

Duval, R. (1998). Signe et objet (I). Trois grandes étapes dans la problématique des rapports entre répresentations et objet. Annales de Didactique et de Sciences Cognitives, 6(6), 139163.

Eco, U. (1979). Trattato di semiotica generale. Milán: Bompiani.

Ernest, P. (1991). The philosophy of mathematics education. Londres: Falmer Press.

Fandiño Pinilla, M. I. (2011). Per una buona didattica è necessario un buon Sapere. Bollettino dei docenti di matematica, 32(62), 51-58.

Flach, P. A., \& Hadjiantonis, A. M. (Compiladores.) (2000). Abduction and Induction: Essays on their Relation and Integration. Dordrecht: Springer.

Font, V., Godino, D. J., \& D'Amore, B. (2007). Ontosemiotic approach of representation in mathematics education. For the learning of mathematics, 27(2), 2-7 y 14.

Gascón, J. (1998). Evolución de la didáctica de las matemáticas como disciplina científica. Recherches en Didactique des Mathématiques, 18(1), 7-33.

Godino, J. D. (2002). Un enfoque ontológico y semiótico de la cognición matemática. Recherches en Didactique des Mathématiques, 22(2-3), 237-284.

Godino, J. D., \& Batanero, C. (1994). Significado institucional y personal de los objetos matemáticos. Recherches en Didactique des Mathématiques, 14(3), 325-355.

Godino, J. D., \& Batanero, C. (1998). Clarifying the meaning of mathematical objects as a priority area of research in mathematics education. En: A. Sierpinska e J. Kilpatrick (Compiladores.) (1998). Mathematics Education as a Research Domain: A Search for Identity. (177-195). Dordrecht: Kluwer A. P.

Godino, J. D., \& Batanero, C. (2016). Implicazioni delle relazioni fra epistemologia e insegnamento della matematica per lo sviluppo curricolare: il caso della combinatoria. $L a$ matematica e la sua didattica, 24(1-2), 19-41. 
Godino, J. D., Batanero, C., \& Roa, R. (2005). An onto-semiotic analysis of combinatorial problems and the solving processes by university students. Educational Studies in Mathematics, 60(1), 3-36.

Hjemslev, L. (1943). Omkring sprogteoriens grundlaggelse. Ed. original en idioma danés. Traducción en inglés: Prolegomena to a Theory of Language. 1961. Madison: University of Winconsin.

Kuhn, T. S. (1957). The Copernican Revolution. Cambridge (Mass): Harvard Univ. Press.

Kutschera, F. Von (1979). Filosofía del lenguaje. Madrid: Gredos.

Lakatos, I. y Musgrave, A. (Compiladores.) (1960). Criticism and the Growth of Knowledge. Cambridge: Cambridge Univ. Press.

Moore, G.E. (1903). The refutation of idealism. Mind. 12, 433-453.

Neisser, U. (1967) Cognitive Psychology. New York: Appleton-Century-Crofts.

Peirce, C. S. 1931-1958. Collected Papers, vols. 1-8. En C. Hartshorne, P. Weiss, A. W. Burks (Compiladores.). Cambridge, MA: Harvard University Press.

Peirce, C. S. (1960). Collected papers of Charles Sanders Peirce (CP, Vol. I-VI). En C. Hartshorne \& P. Weiss (Compiladores.). Cambridge, MA: The Belknap Press of Harvard University Press.

Prediger, S., Bikner-Ahsbahs, A., \& Arzarello, F. (2008). Networking strategies and methods for connecting theoretical approaches: first steps towards a conceptual framework. ZDM Mathematics Education, 38(40), 165-178.

Radford, L. (1998). On Signs and Representations. A Cultural Account. Scientia Paedagogica Experimentalis, 35(1), 277-302.

Radford, L. (2000a). Signs and meanings in students' emergent algebraic thinking: A semiotic analysis. Educational Studies in Mathematics, 42(3), 237-268.

Radford, L. (2000b). Students' processes of symbolizing in algebra. A semiotic analysis of the production of signs in generalizing tasks. En: T. Nakahara y M. Koyama (Compiladores) (2000), Proceedings of the 24th Conference of the International Group for the Psychology of Mathematics Education (PME-24). Hiroshima University, Japón. 4, 81-88

Radford, L. (2001). Factual, Contextual and Symbolic Generalizations in Algebra. En: Marja van den Hueuvel-Panhuizen (Compiladorea) (2001). Proceedings of the 25th Conference of the International Group for the Psychology of Mathematics Education. Freudental Institute, Utrecht University, The Netherlands. 4, 81-88.

Radford, L. (2003). On Culture and Mind. A post-Vygotskian Semiotic Perspective, with an Example from Greek Mathematical Thought. En: M. Anderson, A. Sáenz-Ludlow, S. Zellweger, V. Cifarelli (Compiladores) (2003), Educational Perspectives on Mathematics as Semiosis: From Thinking to Interpreting to Knowing. Ottawa: Legas Publishing. 49-79.

Radford, L. (2006a). Elements of a Cultural Theory of Objectification. Revista Latinoamericana de Investigación en Matemática Educativa, Special Issue on Semiotics. L. Radford y B. D’Amore (Compiladores.), Culture and Mathematical Thinking. 103-129. 
Radford, L. (2006b). Semiótica y educación matemática. Revista Latinoamericana de Investigación en Matemática Educativa, Special Issue on Semiotics. L. Radford y B. D'Amore (Compiladores), Culture and Mathematical Thinking. 7-21.

Radford, L. (2008a). Theories in mathematics education: A brief inquiry into their conceptual differences. ICMI 11 Survey Team 7. The notion and role of theory in mathematics education research. Working paper.

Radford, L. (2008b). Connecting theories in mathematics education: challenges and possibilities. ZDM Mathematics Education. 40, 317-327.

Radford, L. (2011). La evolución de paradigmas y perspectivas en la investigación. El caso de la didáctica de las matemáticas [The evolution of paradigms and perspectives in research. The case of mathematics education]. En: J. Vallès, D. Álvarez y R. Rickenmann (Compiladores) (2011), L'activitat docente: intervenció, innovació, investigació [Teacher's activity: Intervention, innovation, research]. Girona (Español): Documenta Universitaria. 33-49.

Romberg, T. (1988). Necessary ingredients for a theory of mathematics education. En: H. G. Steiner y A. Vermandel (Compiladores) (1988), Foundations and methodology of the discipline Mathematics Education. Proceedings of the $2^{\text {nd }}$ TME. Bielefeld.

Schuppe, W. (1894). Grundriss der Erkenntnistheorie und Logik. Berlin: R. Gaertners.

Sierpinska, A., \& Lerman, S. (1996). Epistemologies of mathematics and of mathematics education. En: A. J. Bishop et al. (Compiladores) (1996), International Handbook of Mathematics Education. (827-876). Dordrecht, HL: Kluwer A. P.

Tall, D. (1991). Reflections on APOS theory in Elementary and Advanced Mathematical Thinking. En: O. Zaslavsky (Compilador) (1991), Proceedings of the 23rd Conference of PME, July 1999. Haifa, Israel. 1, 111-118.

Ullmann, S. (1978). Semántica. Introducción a la ciencia del significado. Madrid: Aguilar. (I edición: 1962).

Vergnaud, G. (1990). La théorie des champs conceptuels. Recherches en Didactique des Mathématiques, (10)2, 133-170.

Wittgenstein, L. (1976). Observaciones sobre los fundamentos de las matemáticas. Madrid: Alianza. 